\title{
On Fitting Rotating Bodies to Exterior Gravitational Fields
}

\author{
M. A. Abramowicz ${ }^{\star}$, J. P. Lasota, and B. Muchotrzeb \\ Institute of Astronomy, Polish Academy of Sciences, Warsaw, Poland
}

\begin{abstract}
Some inequalities have to be satisfied if there is to exist a fluid source for a given exterior gravitational field. In the case of the Kerr solution one of the inequalities presented here is much more restrictive than those obtained by Boyer [1]. However, our conditions do not exclude the possibility of a fluid source for the Kerr spacetimes.
\end{abstract}

\section{Introduction}

Hernandez [2] has shown that for the Kerr metric a relationship is fulfilled between the total mass, $M$, the total angular momentum, $J$, and the quadrupole moment, $Q$ :

$$
Q=J^{2} / M \text {. }
$$

Because of this very special relationship it is believed that the Kerr metric cannot represent correctly the external field of any realistic body [3]. However, the question of the existence of a rigidly rotating, perfect fluid source of the Kerr metric has not yet been answered ${ }^{1}$. This question can arise also in the cases of other exact vacuum solutions representing "rotation of something" such as the Tomimatsu-Sato [5] solution.

In this paper we shall extend a method which has first been used by Boyer [1] to exclude some configurations as sources of the Kerr metric. Boyer has proved that all possible boundaries of rigidly rotating, perfect fluid sources of a Kerr metric, for a given $M$ and $a=J / M$, form at most a two-parameter family of surfaces. The first parameter, $\Omega$, is the angular velocity of the rotating body. The second parameter, $K$, is connected with the polar radius of the body, $r_{p}$, measured in the Boyer-Lindquist coordinates:

$$
r_{p}=M / K+\sqrt{(M / K)^{2}-a^{2}} \text {. }
$$

* Present address: Department of Physics, Stanford University, Stanford, California 94305, USA.

${ }^{-}$According to a recent argument by Roos [12] the method used by Herlt in [4] is incorrect. 
It is clear that the boundary of the body has to be a closed, two-dimensional spacelike surface. Boyer's condition states that for such a surface two inequalities have to be satisfied:

$$
\begin{aligned}
& K \leqq 1 \\
& K \leqq \Omega^{2} a^{2}+3(M \Omega)^{2 / 3}(1-a \Omega)^{4 / 3} .
\end{aligned}
$$

We put $c=G=1$.

In this paper we shall obtain some other inequalities of this type. They follow from Raychaudhuri's equation and from the relativistic virial theorem. In the case of the Kerr metric one of them is much more restrictive than those of Boyer. However, neither Boyer's condition nor any presented in this paper are sufficient to eliminate the possibility of the existence of a rigidly rotating fluid source of the Kerr metric.

We use in this paper the timelike signature +--- .

\section{Definitions and Basic Assumptions}

Let $\eta^{i}$ and $\xi^{i}$ denote two Killing vector fields which exist in the whole spacetime since both interior and exterior solutions are assumed stationary and axially symmetric. The vector field, $\eta^{i}$, is asymptotically unit and timelike and has open trajectories. The vector field, $\xi^{i}$, is spacelike and has closed trajectories $[1,3,6,7,8]$. We shall assume that the four velocity of rotating matter has the form $[3,7]$ :

$$
u^{i}=A\left(\eta^{i}+\Omega \xi^{i}\right) .
$$

We shall also assume that the rotation is rigid i.e. that both shear and expansion scalar of the matter congruence vanish. It is equivalent that the angular velocity, $\Omega$, is constant [6]. The quantity

$$
(1 / A)=\left\{(\eta \eta)+2 \Omega(\eta \xi)+\Omega^{2}(\xi \xi)\right\}^{1 / 2}
$$

is called the redshift factor [3]. According to Boyer [1] it has to be constant on the boundary of the body:

$$
A_{\text {boundary }}=:(1-K)^{-1 / 2}=\text { const. }
$$

Our last assumption is that the stress-energy tensor has the form

$$
T_{k}^{i}=(p+\varepsilon) u^{i} u_{k}-\delta_{k}^{i} p
$$

with $\varepsilon>0$ and $p \geqq 0$ inside the body, Carter [7]. (See [13] for a proof without assumption (2.4).) Using the above assumptions has proved that there exists globally a family $\{\Sigma\}$ of spacelike hypersurfaces orthogonal to the trajectories of the unit, timelike vector field, $n^{i}=e^{\mathscr{U}}\left(\eta^{i}+\tilde{\omega} \xi^{i}\right)$. Here $2 \mathscr{U}:=\left[-\ln \left\{-\varrho^{2} /(\xi \xi)\right\}\right], \varrho^{2}:=$ $(\eta \xi)^{2}-(\eta \eta)(\xi \xi)$ and $\tilde{\omega}:=-(\eta \xi) /(\xi \xi)$. We shall introduce the quantity $\mathscr{R}$ ("distance from the axis of rotation") by the equation

$$
\mathscr{R}:=\varrho A /(u \eta) .
$$


Now, let $h_{k}^{i}$ be the projection tensor on a hypersurface $\Sigma$. Following Bardeen [8] we shall introduce the "physical" three-velocity, $v^{i}$, by the formula

$$
v^{i}\left(1-v^{2}\right)^{-1 / 2}=h_{k}^{i} u^{k} .
$$

Here $v^{2}:=-(v v)=-e^{2 \mathscr{U}}(\xi \xi)(\Omega-\tilde{\omega})^{2}$ is the norm ${ }^{2}$ of $v^{i}$. Notice, that $1-v^{2}=$ $A^{-2} e^{2 \mathscr{U}}$. The quantity $\ell:=\mathscr{R} v$ we shall call the velocity moment. One can show that

$$
\ell=-(u \xi) /(u \eta)=-[(\eta \xi)+\Omega(\xi \xi)] /[(\eta \eta)+\Omega(\eta \xi)] .
$$

The velocity moment $\ell$ is connected with the rotation scalar, $\omega$, for the congruence of trajectories of $u^{i}$ by a formula which has the form very close to its Newtonian counterpart:

$$
\omega^{2}=-(1 / 4)(1-\Omega \ell)^{-2} \mathscr{R}^{-2}\left({ }_{\perp} \nabla^{i} \ell\right)\left({ }_{\perp} \nabla_{i} \ell\right) .
$$

Here ${ }_{\perp} \nabla_{i}$ denotes the covariant derivative on a hypersurface $\Sigma$.

One can show that the total active gravitational mass of the rotating body can be written in the form

$$
M=\int_{I}(\varepsilon+3 p) e^{-\mathscr{U}} d V+2 \Omega J .
$$

Here $I=$ interior $(E=$ exterior), and $d V$ denotes the invariant volume element on $\Sigma$. Equation (2.8) can be called the relativistic virial theorem because in the Newtonian limit it becomes

$$
\mathscr{E}_{\text {grav }}+2 \mathscr{E}_{\text {rot }}+3 \int_{I} p d V=0,
$$

(recall that the Newtonian limit of the 1.h.s. of (2.8) is $M_{0}+\mathscr{E}_{\text {grav }}+\mathscr{E}_{\text {rot }}+\mathscr{E}_{\text {int }}$ where $M_{0}$ is the rest mass and $\mathscr{E}_{\text {int }}$ the internal energy). As far as we know the relativistic virial theorem has never been written in this compact form. However, this form and those known in the literature are only a simple modification of Bardeen's [8] formula for the total mass of a rotating body. Hartle and Sharp [9] were the first who remarked that the Newtonian limit of the relativistic formula for $M$ was connected with the Newtonian virial theorem. To see that (2.8) is equivalent to Bardeen's formula one can use some of Einstein's field equations and then write:

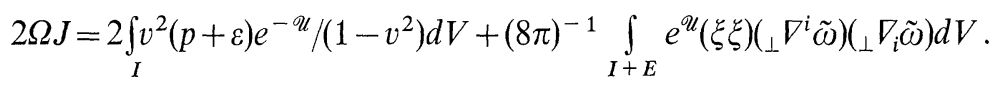

Putting (2.10) into (2.8) one obtains exactly the formula which appears in [8].

Now, let $\lambda^{i}$ be a unit, spacelike vector field which is a linear combination of $\eta^{i}$ and $\xi^{i}$ orthogonal to $u^{i}$. We have $R_{k}^{i} u^{k} \lambda_{i}=0$. Using Ricci the identity one can obtain from the last equation:

$$
{ }_{\perp} \nabla^{i}\left\{e^{-\mathscr{U}} \mathscr{R}^{-2}{ }_{\perp} \nabla_{i} \ell\right\}=0 .
$$

This equation proves that

$$
\int_{I} \omega^{2} e^{-\mathscr{U}} d V=-(1 / 4) \oint_{B} \ell e^{-\mathscr{U}}(1-\Omega \ell)^{-1} \mathscr{R}^{-2}\left({ }_{\perp} \nabla^{i} \ell\right) d B_{i}
$$


where $B$ is the boundary of the body. ${ }^{2}$ It means that the integral which appears on the left hand side of Equation (2.12) can be computed without knowledge of an interval solution.

\section{Basic Inequalities}

Let us project Raychaudhuri's equation on a hypersurface $\Sigma$ and integrate it over the volume of the body. Using the Gauss Theorem and Equations (2.8) and (2.12) one can put the result into the form:

$$
\begin{aligned}
M & -2 \Omega J-(4 \pi)^{-1} \oint_{B}\left({ }_{\perp} \nabla^{i} \ln A\right) e^{-\mathscr{U}} d B_{i} \\
& +(8 \pi)^{-1} \oint_{B} \ell e^{-\mathscr{U}}\left({ }_{\perp} \nabla^{i} \ell\right)(1-\Omega \ell)^{-1} \mathscr{R}^{-2} d B_{i}=0 .
\end{aligned}
$$

We now assert the following inequalities:

$$
\begin{aligned}
& M-2 \Omega J \geqq-(8 \pi)^{-1} \oint_{B} \ell e^{-\mathscr{U}}\left({ }_{\perp} \nabla^{i} \ell\right)(1-\Omega \ell)^{-1} \mathscr{R}^{-2} d B_{i} \\
& 0 \geqq(8 \pi)^{-1} \oint_{B} \ell e^{-\mathscr{U}}\left({ }_{\perp} \nabla^{i} \ell\right)(1-\Omega \ell)^{-1} \mathscr{R}^{-2} d B_{i} \\
& \Omega J \geqq(16 \pi)^{-1} \oint_{B} \tilde{\omega}(\xi \xi) e^{\mathscr{U}}\left({ }_{\perp} \nabla^{i} \tilde{\omega}\right) d B_{i} .
\end{aligned}
$$

(3.2) follows from (3.1) and the fact that $\ln A=\int_{B}^{p} d p /(p+\varepsilon(p))+$ const (the last equality is a consequence of the equations of motion $\nabla_{i} T^{i j}=0$ ), because the pressure is zero on the surface of the body and nonnegative inside. (3.3) is implied by (2.12) and (3.4) is obtained by using (2.10) and Equation (a) of the Appendix.

Inequality (3.2) becomes in the Newtonian limit the well-known Poincare condition

$$
2 \pi M \geqq \Omega^{2} V .
$$

Where $V$ is the volume of the body. If it not satisfied then the body cannot be stationary and has to expand [10]:

$$
\int_{I} \dot{\theta} e^{-\mathscr{u}} d V \geqq 0,
$$

where $\dot{\theta}=u^{k} \nabla_{k} \nabla_{i} u^{i}$ is the change of the expansion rate and $\nabla_{i}$ denotes the covariant derivative in the whole spacetime.

From Equation (2.7) it follows that if (3.3) is not satisfied then either there exists a region inside the surface given by Equation (2.3) in which $\varrho \leqq 0$ [it means physically that there exists an event horizon $(\varrho=0)$ inside the body], or that the gradient of the velocity moment $\nabla_{i} \ell$, is a timelike vector. Both these possibilities are absurd. We are not successful in the elucidation of the physical meaning of

\footnotetext{
2 Equation (2.11) can be integrated in a special coordinate system in which $x:=(\ell / \Omega)^{1 / 2}$ is one spatial coordinate. One then obtains $x \varrho^{2} \mathscr{R}^{-3}=g_{x x} / g_{y y}$. The last two equations reduce the number of independent components of $g_{t k}$ to three. The second spatial coordinate, $y$, is chosen in such a way that $g_{\imath y}=0$.
} 
Fig. 1. The region of admissible values of $\Omega$ and $K$ for $a / M=0.001 . K(a)=0.67$, $M \Omega(a)=0.000, K(b)=0.68, M \Omega(b)=0.117$, $K(c)=1.00, M \Omega(c)=0.192$

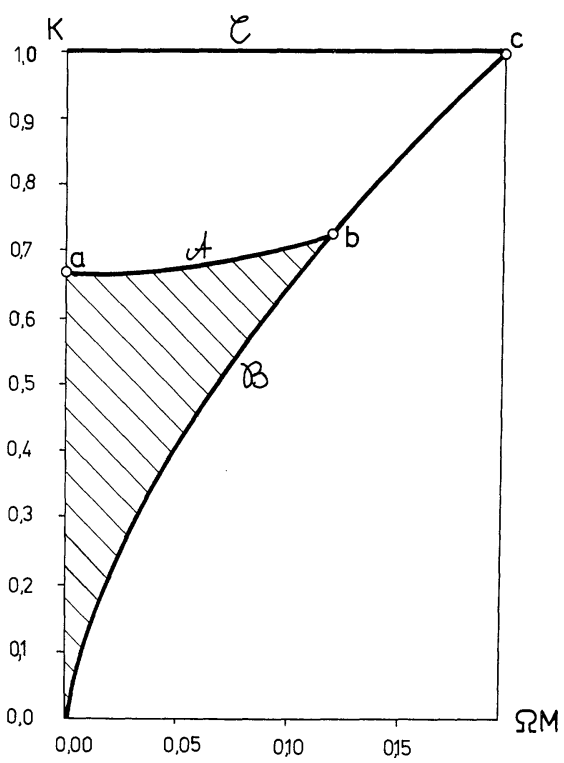

inequality (3.4). In the Newtonian limit it becomes the trivial condition $J \Omega \geqq 0$ and it seems to be less restrictive than the condition $\Omega \geqq \max (\tilde{\omega})$ which, as it is generally believed [3], has to be fulfilled in any realistic body. As in all the inequalities (3.4), (3.5), (3.6) only surface integrals appear, we can check them with no knowledge of the interior solution.

\section{Application to the Kerr Solution}

In the case of the Kerr solution we have in Boyer-Lindquist coordinates:

$$
\begin{aligned}
& (\eta \eta)=1-2 M /\left(r^{2}+a^{2} \cos ^{2} \theta\right), \\
& (\eta \xi)=2 M r a \sin ^{2} \theta /\left(r^{2}+a^{2} \cos ^{2} \theta\right), \\
& (\xi \xi)=-\sin ^{2} \theta\left\{r^{2}+a^{2}+2 M r a^{2} \sin ^{2} \theta /\left(r^{2}+a^{2} \cos ^{2} \theta\right)\right\} .
\end{aligned}
$$

An application of inequalities (1.3), (1.4), and (3.2), (3.3), (3.4) in the case of $a / M=0.001$ is shown in Figure 1 and in the case of $a / M=1$ in Figure 2 . In these figures the curves $\mathscr{B}$ represent Boyer's condition (1.4) and the lines $\mathscr{C}$ represent Boyer's condition (1.3). The curves $\mathscr{A}$ are calculated numerically from inequality (3.3). Inequalities (3.2) and (3.4) are satisfied in the region between $\mathscr{A}$ and $\mathscr{B}$ and they do not give additional restrictions. One can observe that the region of admissible values of the parameters $K$ and $\Omega$ increases for $0 \leqq a / M \leqq 1$ with increasing $a / M$. However, when $a / M>1$ we have two additional restrictions which follow from the relativistic virial theorem and from the condition that the polar radius, $r_{p}$, has to be real:

$$
\Omega \leqq 1 / 2 a, \quad K \leqq M / a .
$$




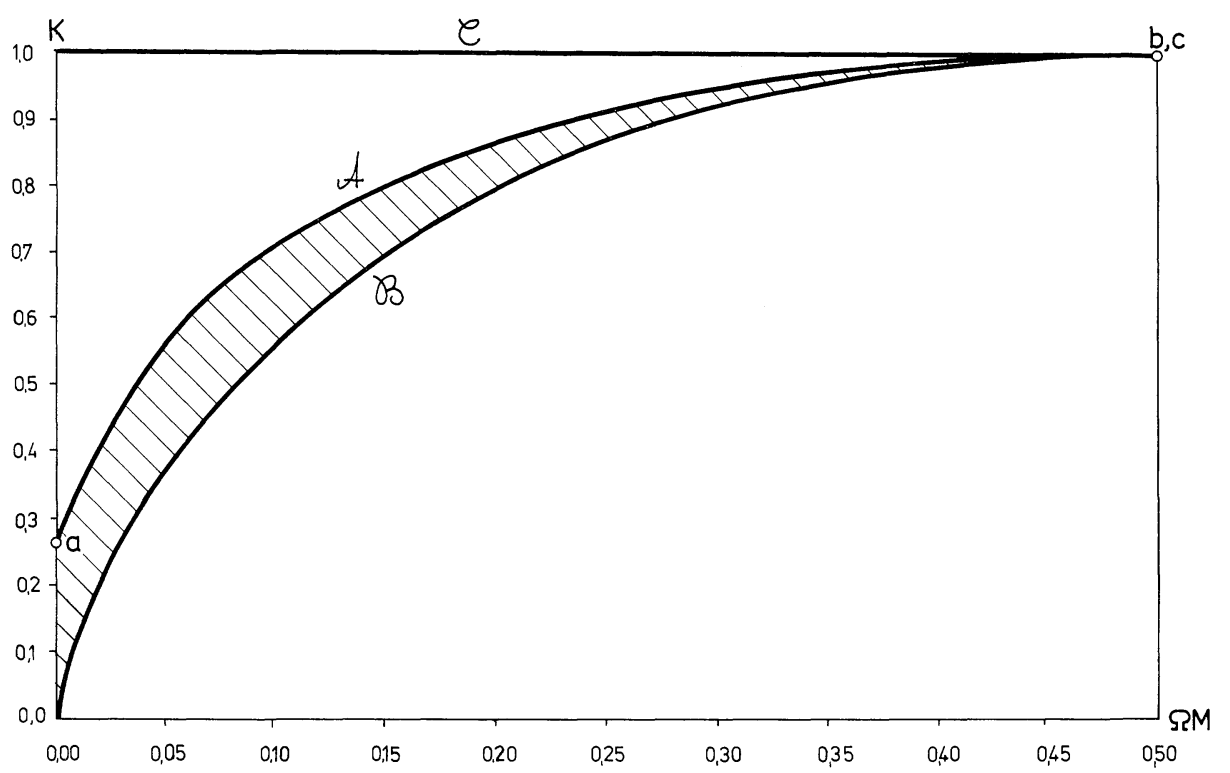

Fig. 2. The region of admissible values of $\Omega$ and $K$ for $a / M=1 . K(a)=0.22, M \Omega(a)=0.00, K(b)=K(c)=$ $1.00, M \Omega(b)=M \Omega(c)=0.50$. Point $b$ represents the most relativistic material source of the Kerr metric

This means that for very great $a / M, K \approx 0$ and $\Omega \approx 0$ are the only possible values of the parameter $K$ and $\Omega$. In other words configurations (if they exist) which are the sources of the Kerr metric and have $a / m \gg 1$ are in a sense nearly Newtonian. The most relativistic source of the Kerr metric is characterized by the following values:

$$
a=M, \quad K=1, \quad \Omega M=1 / 2 .
$$

\section{Conclusions}

Although our condition (3.3) is much more restrictive than those of Boyer, it is not sufficient to eliminate the possibility of the existence of a fluid source of the Kerr spacetimes even for very great values of $a / M$.

It will probably be interesting to apply our conditions to some other vacuum solutions of the Einstein field equations.

\section{Appendix}

We shall present here all the Einstein field equations which have been used. They are $[14,15]$ :

$$
\begin{aligned}
& R_{k}^{i} n_{i} \xi^{k}=\frac{1}{2}{ }_{\perp} \nabla^{i}\left\{e^{\mathscr{U}}(\xi \xi)_{\perp} \nabla_{i} \tilde{\omega}\right\}=32 \pi e^{-\mathscr{U}} \ell(p+\varepsilon) /(1-\Omega \ell), \\
& R_{k}^{i} n_{i} \eta_{-}^{k}={ }_{\perp} \nabla^{i}\left\{e^{-\mathscr{U}}{ }_{\perp} \nabla_{i} \mathscr{U}+\frac{1}{2}(\eta \xi) e^{\mathscr{U}}{ }_{\perp} \nabla_{i} \tilde{\omega}\right\} \\
& =4 \pi e^{-\mathscr{U}}(\varepsilon+3 p)+8 \pi e^{-\mathscr{U}} \ell \Omega(p+\varepsilon) /(1-\Omega \ell), \\
& e^{-\mathscr{U}} R_{k}^{i} u_{i} u^{k}={ }_{\perp} \nabla^{i}\left\{e^{-\mathscr{U}}{ }_{\perp} \nabla_{i} \ln A\right\}+2 \omega^{2} e^{-\mathscr{U}}=4 \pi(\varepsilon+3 p) e^{-\mathscr{U}} .
\end{aligned}
$$

The last one is Raychaudhuri's equation projected on a hypersurface $\Sigma$. 
We would like to thank Professor Andrzej Trautman for his kind interest in this work, and Maciej Kozlowski and Alexander Czerny for helping us with the preparation of the computational programme. We are also greatly indebted to Professors J. Ehlers and W. Kundt for comments and suggestions.

\section{References}

1. Boyer, R.H.: Proc. Camb. Phil. Soc. 61, 527 (1965)

2. Hernandez, W.: Phys. Rev. 159, 1070 (1967)

3. Thorne, K.S.: In Gravitation and Cosmology, Proceedings of the International School of Physics, Enrico Fermi XLVII (ed. R. K. Sachs). New York: Academic Press 1971

4. Herlt, E.: Ann. Phys. 7, 178 (1970)

5. Tomimatsu, A., Sato, H.: Phys. Rev. Letters 29, 1344 (1972)

6. Boyer, R.H.: Proc. Camb. Phil. Soc. 62, 495 (1966)

7. Carter, B.: J. Math. Phys. 10, 70 (1969)

8. Bardeen, J. M.: Ap. J. 162, 71 (1970)

9. Hartle, J. B., Sharp, D. H.: Ap. J. 147, 317 (1967)

10. Abramowicz, M.A.: Acta Astronom. 21, 449 (1971)

11. Bardeen, J. M., Wagoner, R. V.: Ap. J. 167, 359 (1971)

12. Roos, W.: Diplom-Thesis, Hamburg University (1974)

13. Greene, R. D., Schucking, E. L., Visheshvara,C.V.: The Rest Frame in Stationary Spacetimes with Axial Symmetry, Preprint

14. Abramowicz, M.A.: Some Special Vector Fields in Stationary and Axial Symmetric Spacetimes. Preprint (1973)

15. Bardeen, J. M.: In Black-Holes, proceedings of the Les Houches Summer School 1972 (eds. C. and B.S.DeWitt). New York: Gordon and Breach 1973

Communicated by J. Ehlers

Received August 29, 1974; in revised form November 10, 1975 
